\title{
Determining large deflections in rectangular combined loaded cantilever beams made of non-linear Ludwick type material by means of different arc length assumptions
}

\author{
IBRAHIM EREN \\ Yildiz Technical University, Mechanical Engineering Department, 34349, \\ Besiktas, Istanbul, Turkey \\ e-mail: er@yildiz.edu.tr
}

MS received 12 November 2007; accepted 20 January 2008

\begin{abstract}
In this study, large deflection of cantilever beams of Ludwick type material subjected to a combined loading consisting of a uniformly distributed load and one vertical concentrated load at the free end was investigated. In calculations, both material and geometrical non-linearity have been considered. Horizontal and vertical deflections magnitudes were calculated throughout Euler-Bernoulli curvature-moment relationship assuming different arc lengths. Vertical deflections were calculated by using Runge-Kutta method. More simple and easily understandable results have been obtained compared to the previous studies about the issue and compatible values have been obtained for most of the compared values.
\end{abstract}

Keywords. Large deflections; material non-linearity; geometrical non-linearity.

\section{Introduction}

Large deflections under variable loads in bearer systems is a popular subject on which many studies were conducted. Due to the importance of this subject, studies are being done on the issue. In many cases encountered in different engineering issues, results are adequately approximate. However, well known curvature is not linear in bending and thus, real material does not have linear stress-strain relationship. When this fact is considered, deflections can not be determined by analytical methods, instead numerical and approximate methods should be employed. Large deflection in uniform and non-uniform, concentrated and combined loaded linear elastical cantilever beams have been investigated in earlier studies (Bisshopp \& Drucker 1945; Scott et al 1955; Lau 1982; Rao \& Rao 1986; Baker 1993; Lee et al 1993; Frisch-Fay 1962; Fertis 1999). Prathap and Varadan (1976) had calculated large deflections in cantilever beams made of non-linear Ramberg-Osgood type material on which concentrated load effected on the free end. Same problem had been solved for cantilever beams on which moment effected on the free end by Varadan \& Joseph (1987). Large deflections in Ludwick type non-linear cantilever beams on which concentrated load effected on the free end, had been investigated by Lewis \& Monasa (1981). Lewis and Monasa (1982) had solved the same problem for cantilever beams on which moment effected on the free end. 


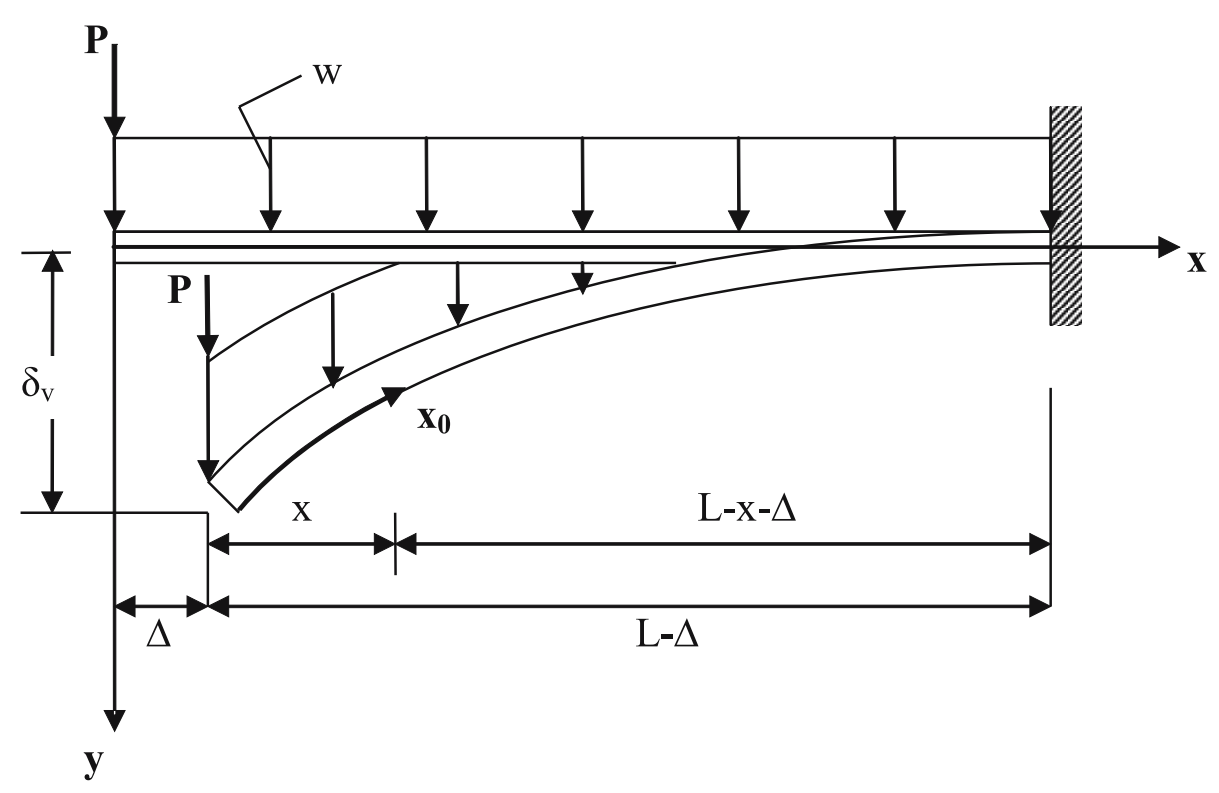

Figure 1. Cantilever beam subjected to combined load.

Lo and Gupta (1978) had calculated large deflections in rectangular beams by assuming logarithmical material stress-strain relationship beyond elastic limit. Lee (2002) had calculated large deflections in cantilever beams made of Ludwick type non-linear material on which uniformly distributed load and concentrated load effected on the free end.

\section{Combined loaded cantilever beam}

In this study, large deflections have been calculated in non-linear Ludwick type rectangular combined loaded cantilever beams by means of different arc length assumptions, as shown in figure $1 . P$ is the concentrated load effecting on the free end, $L$ is the length of cantilever beam, $w$ is the uniformly distributed load of intensity, $x_{o}$, arc length, $\Delta$, horizontal deflection magnitude, $\delta v$, vertical deflection magnitude at the free end. Mathematica 5.2 software has been employed for calculations.

\section{Formulation and solution}

Moment expression, depending on uniformly distributed load of intensity $w$, is given below for figure 1 .

$$
M=\frac{w x}{2} x_{0}+P x .
$$

Assumptions made by Fertis for arc length and horizontal deflection functions are given below:

$$
x_{0}=x+\Delta(x), \text { Fertis (1999), }
$$




$$
\begin{aligned}
& \Delta(x)=\Delta, \text { Fertis (1999), } \\
& \Delta(x)=\frac{\Delta x}{(L-\Delta)}, \text { Fertis (1999). }
\end{aligned}
$$

If (2) and (3) are written in the moment expression,

$$
M=\frac{w x}{2}(x+\Delta)+P x
$$

is obtained.

$$
P=w L, \text { Lee (2002) }
$$

is an assumption.

$$
\sigma=B \varepsilon^{\frac{1}{n}},(\text { Lewis \& Monosa 1982). }
$$

This expression is stress-strain relationship for Ludwick type material. Here, $\sigma$ is stress, $\varepsilon$ is strain, $B$ and $n$ is constant depending on material properties.

Euler-Bernoulli curvature-moment relationship is shown below:

$$
\kappa=\frac{y(x)}{\left(1+\left(y^{\prime}(x)\right)^{2}\right)^{\frac{3}{2}}}=\frac{M^{n}}{K_{n}}, \text { (Lewis \& Monosa 1981). }
$$

Here, $\kappa$ is the curvature and $K_{n}$ is a constant depending on material properties

$$
K_{n}=\frac{n^{n} b^{n} h^{2 n+1} B^{n}}{2^{n+1}(1+2 n)^{n} P^{n}},(\text { Lewis \& Monosa 1981) }
$$

$b$ is the width and $h$ is the height of the rectangular cross section. If moment expression found for the first arc length assumption and previous equations are employed, curvature equation can be stated as below:

$$
\kappa=\frac{L^{n+1}}{K_{n}} \times \frac{1}{L}\left(\frac{x}{2 L}\left(\frac{x}{L}+\frac{\Delta}{L}+2\right)\right)^{n},
$$

curvature equation is written according to $\frac{L^{n+1}}{K_{n}}$ dimensionless value.

If both sides of the curvature equation in (8) are integrated;

$$
\int \kappa d x+C_{1}=\frac{y^{\prime}(x)}{\left(1+\left(y^{\prime}(x)\right)^{2}\right)^{\frac{1}{2}}}
$$

is obtained.

$$
\begin{aligned}
& \int \kappa d x+C_{1}=G, \text { Fertis (1999) } \\
& y^{\prime}(x)=\frac{G}{\left(1-(G)^{2}\right)^{\frac{1}{2}}}, \text { Fertis (1999) } \\
& \frac{x}{L}=\bar{x}, \frac{\Delta}{L}=\delta_{h}
\end{aligned}
$$


If these equations are converted to dimensionless units and thus,

$$
G=\frac{2^{-n} \frac{L^{n+1}}{K_{n}} \bar{x}\left(\bar{x}\left(\bar{x}+\left(2+\delta_{h}\right)\right)\right)^{n}\left(1+\frac{\bar{x}}{2+\delta_{h}}\right)_{2}^{-n} F 1\left[1+n,-n ; 2+n ;-\frac{\bar{x}}{2+\delta_{h}}\right]}{(1+n)}+C_{1}
$$

is obtained.

$$
\bar{x}=1-\delta_{h} ;
$$

$y^{\prime}\left(1-\delta_{h}\right)$ value is taken as zero as a boundary condition, $C_{1}$ integration constant is obtained as shown below:

$$
C_{1}=-\frac{\left(\frac{3}{2}\right)^{n} \frac{L^{n+1}}{K_{n}}\left(1-\delta_{h}\right)^{n+1}\left(1+\frac{1-\delta_{h}}{2+\delta_{h}}\right)_{2}^{-n} F 1\left[1+n,-n ; 2+n ;-\frac{1-\delta_{h}}{2+\delta_{h}}\right]}{(1+n)}
$$

Finally,

$$
\begin{aligned}
G= & \frac{2^{-n} \frac{L^{n+1}}{K_{n}} \bar{x}\left(\bar{x}\left(\bar{x}+\left(2+\delta_{h}\right)\right)\right)^{n}\left(1+\frac{\bar{x}}{2+\delta_{h}}\right)_{2}^{-n} F 1\left[1+n,-n ; 2+n ;-\frac{\bar{x}}{2+\delta_{h}}\right]}{(1+n)} \\
& \pm \frac{\left(\frac{3}{2}\right)^{n} \frac{L^{n+1}}{K_{n}}\left(1-\delta_{h}\right)^{n+1}\left(1+\frac{1-\delta_{h}}{2+\delta_{h}}\right)_{2}^{-n} F 1\left[1+n,-n ; 2+n ;-\frac{1-\delta_{h}}{2+\delta_{h}}\right]}{(1+n)}
\end{aligned}
$$

is obtained.

Arc length equation is,

$$
\begin{aligned}
& \int_{0}^{(L-\Delta)} \sqrt{\left(1+\left(y^{\prime}(x)\right)^{2}\right)}=L,(\text { Lewis \& Monosa 1982) } \\
& \int_{0}^{(L-\Delta)} \sqrt{\left(1+\left(\frac{G}{\left(1-(G)^{2}\right)^{\frac{1}{2}}}\right)^{2}\right)}=L
\end{aligned}
$$

If $G$ expression is written in the arc length equation and 3 to 1 rule of Simpson is integrated, then $\delta_{h}$ horizontal deflection could be calculated by finding root of the equation obtained with Newton method. This process is repeated depending on different values of $n$ and dimensionless $L^{n+1} / K_{n}$ ratio.

If, $y\left(1-\delta_{h}\right)=0$ boundary condition that can be written from (13) and (17) considered, $y(\bar{x})$ interpolation function is obtained by the help of Runge-Kutta method. In this function, $y(0)$ value for $\bar{x}=0$ gives the vertical deflection at the free end of the beam as a dimensionless $\delta_{v} / L$ value.

New curvature expression is written as below if the previous problem is solved by employing $x_{0}$ arc length assumption which is selected using different horizontal deflection functions stated in (2) and (4).

$$
\kappa=\frac{L^{n+1}}{K_{n}} \times \frac{1}{L}\left(\frac{x}{2 L}\left(\frac{x}{L}+\frac{x}{L} \frac{\frac{\Delta}{L}}{\left(1-\frac{\Delta}{L}\right)}+2\right)\right)^{n}
$$


$G$ is found as below when dimensionless conversion made, previous curvature and appropriate equations are employed:

$$
\begin{aligned}
G= & \frac{2^{-n} \frac{L^{n+1}}{K_{n}} \bar{x}\left(-\frac{\bar{x}\left(\bar{x}-2\left(-1+\delta_{h}\right)\right)}{\left(-1+\delta_{h}\right)}\right)^{n}\left(1+\frac{\bar{x}}{2-2 \delta_{h}}\right)^{-n}}{(1+n)} \\
& \times{ }_{2} F 1\left[1+n,-n ; 2+n ;-\frac{\bar{x}}{2-2 \delta_{h}}\right]+C_{1}
\end{aligned}
$$

When $y^{\prime}\left(1-\delta_{h}\right)=0$ boundary condition is employed in $\bar{x}=1-\delta_{h}, C_{1}$ integration constant becomes,

$$
\begin{aligned}
C_{1}= & -\frac{2^{-n} \frac{L^{n+1}}{K_{n}}\left(1+\frac{1-\delta_{h}}{2-2 \delta_{h}}\right)^{-n}\left(1-\delta_{h}\right)\left(-\frac{\left(1-\delta_{h}\right)\left(1-2\left(-1+\delta_{h}\right)-\delta_{h}\right)}{\left(-1+\delta_{h}\right)}\right)^{n}}{(1+n)} \\
& \times{ }_{2} F 1\left[1+n,-n ; 2+n ;-\frac{\left(1-\delta_{h}\right)}{\left(2-2 \delta_{h}\right)}\right]
\end{aligned}
$$

thus,

$$
\begin{aligned}
G= & \frac{2^{-n} \frac{L^{n+1}}{K_{n}} \bar{x}\left(-\frac{\bar{x}\left(\bar{x}-2\left(-1+\delta_{h}\right)\right)}{\left(-1+\delta_{h}\right)}\right)^{n}\left(1+\frac{\bar{x}}{2-2 \delta_{h}}\right)^{-n}}{(1+n)} \times{ }_{2} F 1\left[1+n,-n ; 2+n ;-\frac{\bar{x}}{2-2 \delta_{h}}\right] \\
& \pm \frac{2^{-n} \frac{L^{n+1}}{K_{n}}\left(1+\frac{1-\delta_{h}}{2-2 \delta_{h}}\right)^{-n}\left(1-\delta_{h}\right)\left(-\frac{\left(1-\delta_{h}\right)\left(1-2\left(-1+\delta_{h}\right)-\delta_{h}\right)}{\left(-1+\delta_{h}\right)}\right)^{n}}{(1+n)} \\
& \times{ }_{2} F 1\left[1+n,-n, 2+n,-\frac{\left(1-\delta_{h}\right)}{\left(2-2 \delta_{h}\right)}\right]
\end{aligned}
$$

is obtained.

$\delta_{h}$ horizontal deflection values are calculated by finding root of the arc length equation, which is written according to $G$ expression. This calculation is made by utilising Simpson rule and Newton method. This process is repeated depending on different values of $n$ and dimensionless $L^{n+1} / K_{n}$ ratio.

If, $y\left(1-\delta_{h}\right)=0$ boundary condition that can be written from (13) and (17) considered, $y(\bar{x})$ interpolation function is obtained by the help of Runge-Kutta method. In this function, $y(0)$ value for $\bar{x}=0$ gives the vertical deflection at the free end of the beam as a dimensionless $\delta_{v} / L$ value.

\section{Results and discussion}

In tables 1 and 2, dimensionless horizontal and vertical deflection values, which were calculated for two different $x_{o}$ arc length assumptions, are tabulated depending on $L^{n+1} / K_{n}$ ve $n$ values. Besides, for $n=2 \cdot 16$ as given in (Lewis \& Monosa 1982) (7) equation, deflection in cantilever beams made of Ludwick type non-linear annealed copper material which were loaded as shown in figure 1, has been given in comparison with the result of Lee (2002). 


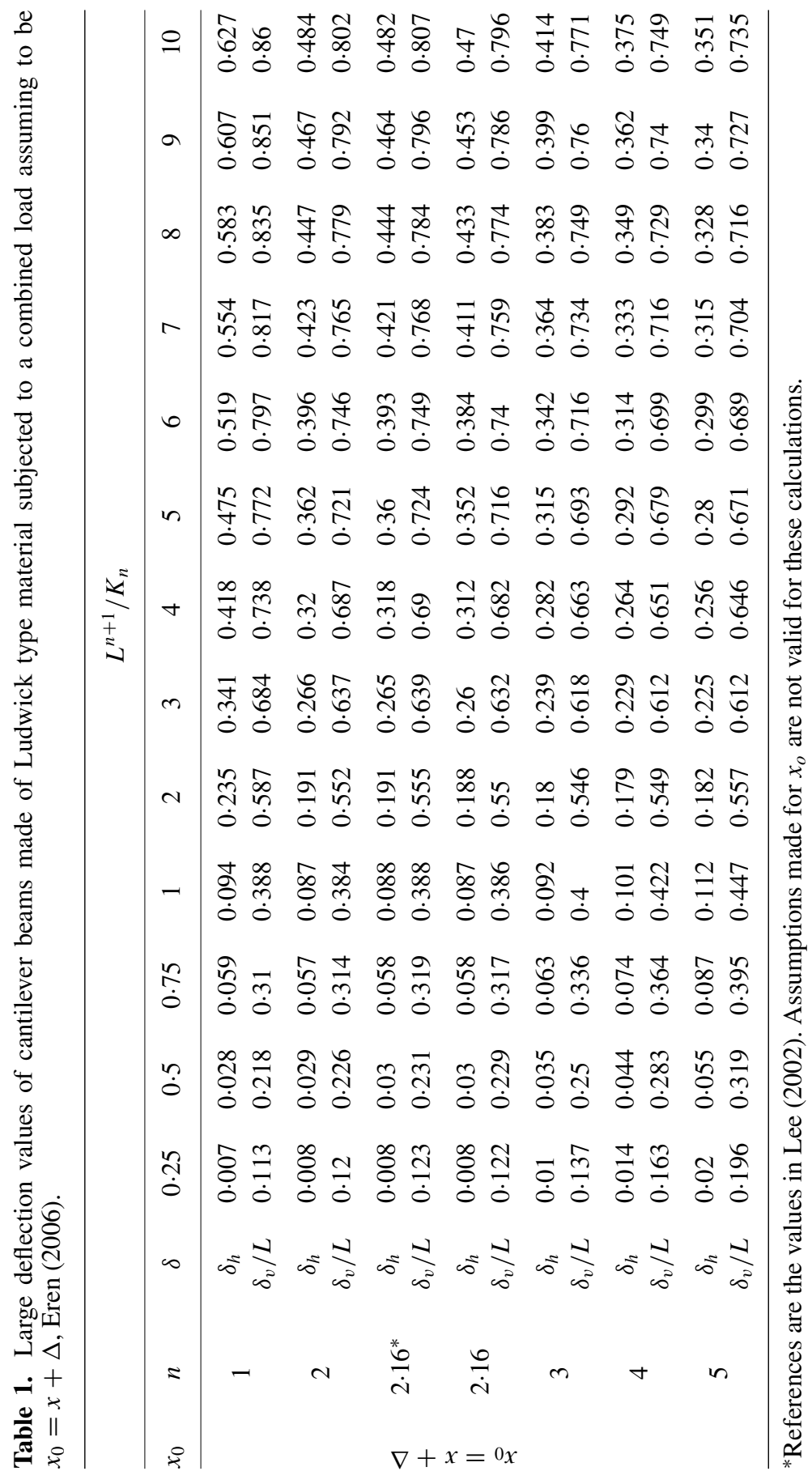




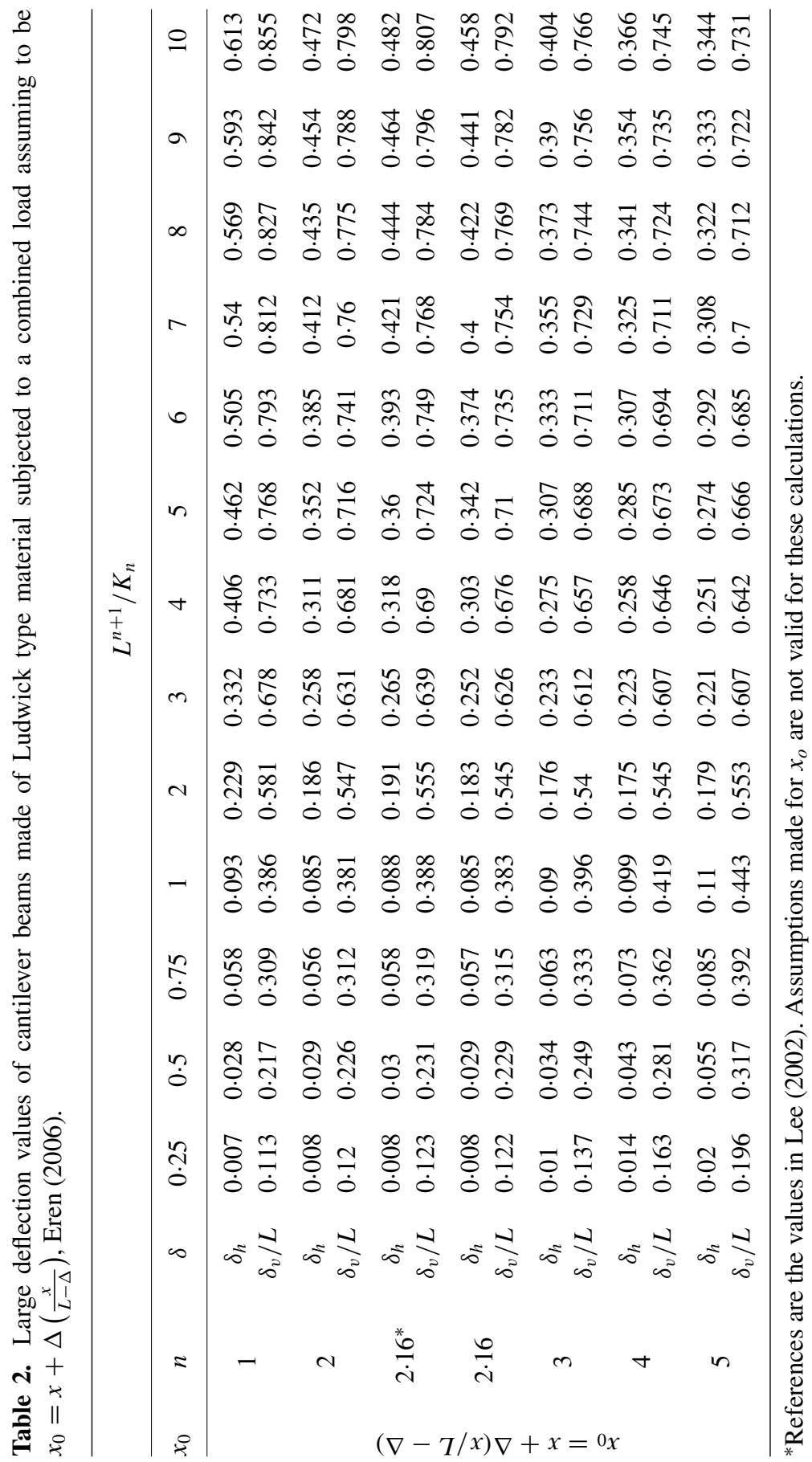




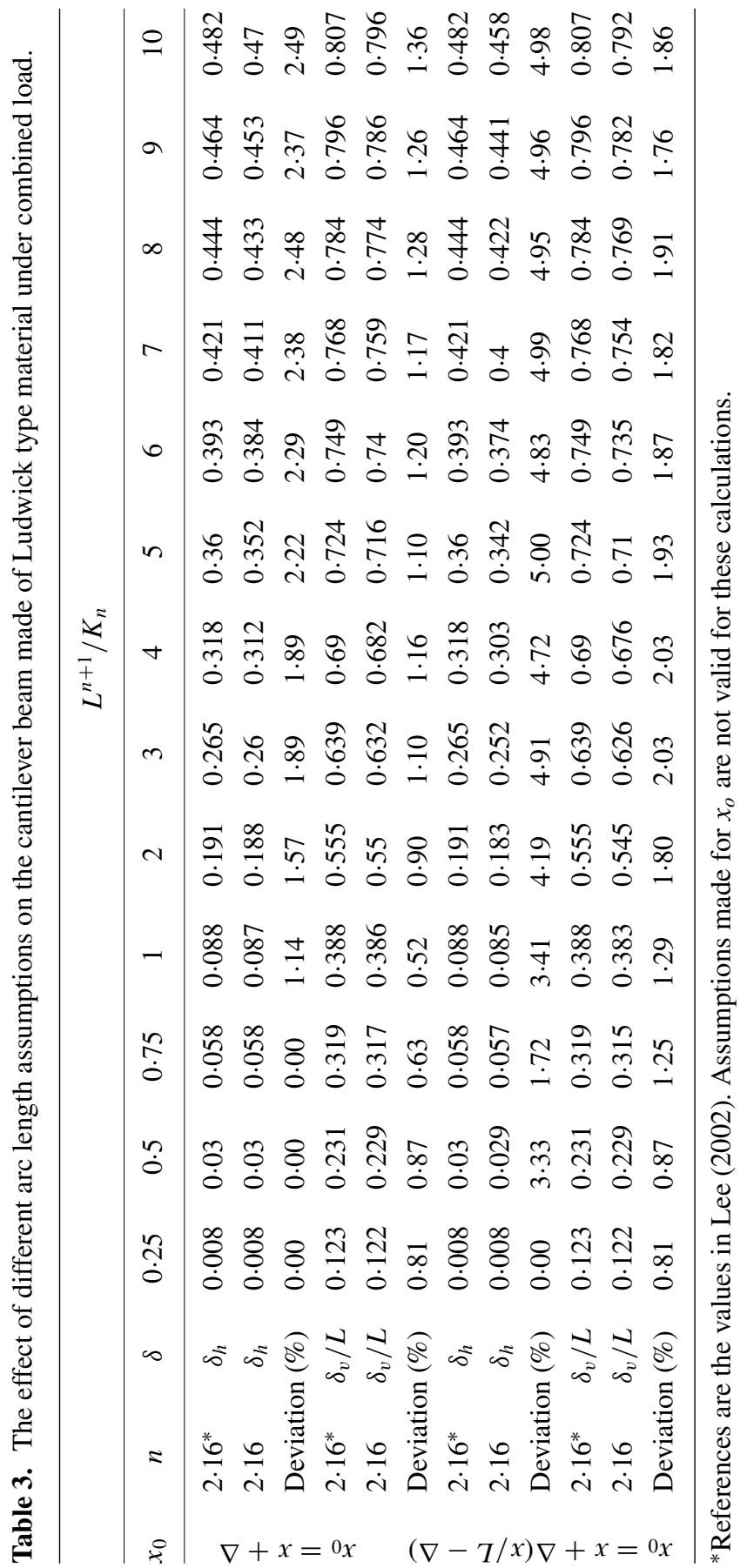




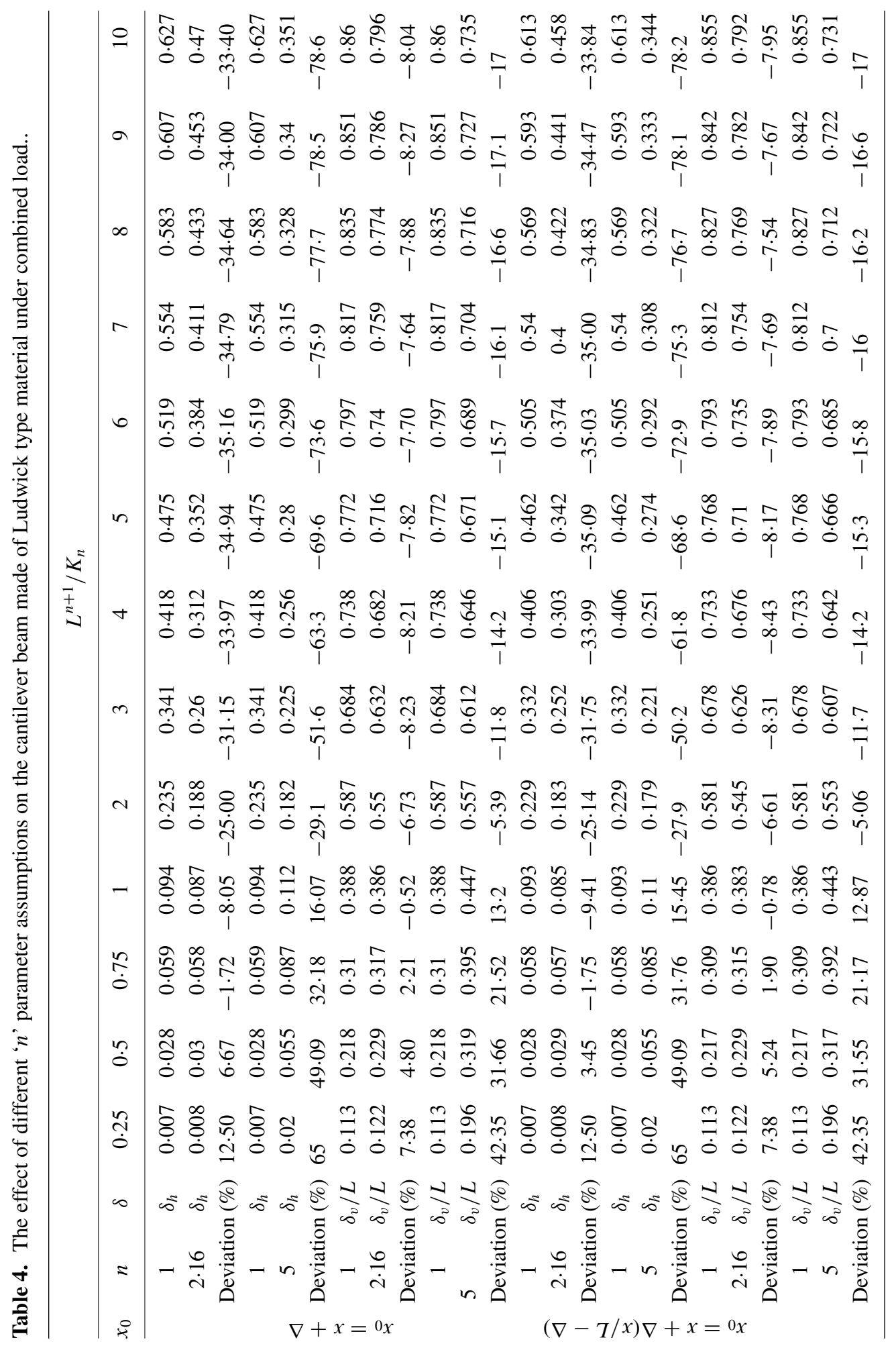


When table 3 is analysed, deviation between horizontal deflections is in the $0 \%$ and $2.49 \%$ range depending on $\frac{L^{n+1}}{K_{n}}$ dimensionless value. This range is calculated for Ludwick type annealed copper material if $x_{0}=x+\Delta$ assumption is made for horizontal deflection. $n=2.16$ for this condition as stated in reference Lee (2002). If we analyse the vertical deflection at the free end deviation from the reference Lee (2002) is in the $0.52-1.52 \%$ range. If we assume that $x_{0}=x+\Delta\left(\frac{x}{L-\Delta}\right)$ then, horizontal deflection deviation is between 0 and $5 \%$ and vertical deflection deviation is between 0.81 and $2.03 \%$ compared to the reference Lee (2002) values.

In table 4, deflection values for different $n$ and $x_{o}$ values and deviations from deflection values for $n=1$ (linear) are shown. Here, deviation is not a mistake instead, a statement showing the change in percentage. In table 4 , for $\frac{L^{n+1}}{K_{n}}$ values where deviation is positive, vertical and horizontal deflections increase with increasing $n$ and if deviation is negative vertical and horizontal deflections decrease with increasing $n$.

\section{Conclusion}

It should be seen that, deviation (\%) for horizontal deflection values is greater than vertical deflection values for both $x_{o}$ assumption. It can be concluded that $x_{0}=x+\Delta$ assumption is more applicable for these calculations because this assumption gives smaller deviations than $x_{o}$ assumption for vertical and horizontal deflection values and also it gives smaller values than the values obtained by Lee (2002).

If $x_{0}$ arc length is employed with close assumptions as shown previously, deflection calculation is simplified. In such cases, $y^{\prime}(x)$ expression could be obtained by making $x_{0}$ assumption and employing curvature. Thus, deflection calculations become possible for different loading and joint structures by employing the mentioned solution method which is also employed for solving many other problems.

Bottom line, method employed by Fertis (1999) which is based on 2 different $x_{0}$ arc length gives much more simplified, easier and understandable solution than the method employed by Lee (2002) for calculation of large deflections in combined loaded, cantilever beams that has Ludwick type stress-strain relationship.

\section{List of symbols}

$b \quad$ width of the rectangular cross section

$B \quad$ Constant depending on Ludwick type material properties

$h$ height of the rectangular cross section

$K_{n} \quad$ Constant depending on Ludwick type material properties

$L \quad$ Length of cantilever beam

$M \quad$ Bending moment

$n \quad$ Constant depending on Ludwick type material properties

$P \quad$ Concentrated load

$w \quad$ Uniformly distributed load of intensity

$x_{o} \quad$ Arc length

$\Delta \quad$ Horizontal deflection magnitude

$\Delta(x)$ Horizontal deflection function 
$\delta v \quad$ Vertical deflection magnitude at the free end

$\sigma \quad$ Stress

$\varepsilon \quad$ Strain

$\kappa \quad$ Curvature

\section{References}

Baker G 1993 On the large deflections of non-prismatic cantilevers with a finite depth. Comput. Struct. 46: 365-370

Bisshopp K E, Drucker D C 1945 Large deflections of cantilever beams. Q. Appl. Math. 3: 272-275

Eren I, 2006 Some new solutions on large deflections of beams (in Turkish). PhD Dissertation, Institute of Science, Yildiz Technical University, Istanbul

Fertis D G 1999 Nonlinear Mechanics, (New York: CRC Pres)

Frisch-Fay R 1962 Flexible Bars, (London: Butter Worths)

Lau J H 1982 Large deflections of beams with combined loads. J. Eng. Mech. 108: 180-185

Lee B K, Wilson J F, Oh S J 1993 Elastica of cantilevered beams with variable cross sections. Int. J. Non-Linear Mech. 28: 579-589

Lee K 2002 Large deflections of cantilever beams of non-linear elastic material under a combined loading. Int. J. Non-Linear Mech. 37: 439-443

Lewis G, Monosa F 1981 Large deflections of cantilever beams of non-linear materials. Comput. Strct. 14: $357-360$

Lewis G, Monosa, F 1982 Large deflections of cantilever beams of non-linear materials of the Ludwick type subjected to an end moment. Int. J. Non-Linear Mech. 17: 1-6

Lo C C, Gupta, S D 1978 Bending of a non-linear rectangular beam in large deflection. J. Appl. Mech. 45: 213-215

Prathap G, Varadan T K 1976 The inelastic large deformation of beams, J. Appl. Mech. 43: 689-690

Rao B N, Rao G V 1986 On the large deflection of cantilever beams with end rotational load. Z. AngewMath. Mech. 66: 507-509

Scott E J, Carver D R, Kan M 1955 On the linear differential equation for beam deflection. J. Appl. Mech. 22: 245-248

Varadan T L, Joseph D 1987 Inelastic finite deflections of cantilever beams. J. Aeronaut. Soc. India 39: $39-41$ 